\title{
Image Enhancement based on Stationary Wavelet Transform, Integer Wavelet Transform and Singular Value Decomposition
}

\author{
Neena K. A \\ Federal Institute of Science and \\ Technology, Angamaly \\ Kerala \\ India
}

\author{
Aiswariya Raj \\ Assistant Professor \\ Federal Institute of Science and \\ Technology, Angamaly \\ Kerala \\ India
}

\author{
Rajesh Cherian Roy \\ Associate Professor \\ Federal Institute of Science and \\ Technology, Angamaly \\ Kerala \\ India
}

\begin{abstract}
In this paper, the image enhancement technique with respect to resolution and contrast has been proposed. The proposed method is based on bi cubic interpolation, stationary wavelet transform, discrete wavelet transform and singular value decomposition. In the proposed technique stationary wavelet transform decomposes the input image into sub bands having different frequency coefficients. The high frequency coefficients have been multiplied with the orthogonal matrix (hanger and aligner) coefficients, obtained using singular value decomposition of low resolution input image. Resulting sub bands are further added with the high frequency sub bands obtained using stationary wavelet transform. Then, the low resolution input image and high frequency sub band images are interpolated using bi cubic interpolation. Inverse integer wavelet transform has been used to combine all these sub images. Image equalization is done on this image using singular value equalization. The proposed technique is tested on different satellite images. The experimental results show the proposed method gives good results over conventional methods.
\end{abstract}

\section{General Terms}

Bi_cubic interpolation, Stationary wavelet transform (SWT), Singular value decomposition (SVD), Integer wavelet transform (IWT), Inverse Integer Wavelet Transform (IIWT)

\section{INTRODUCTION}

In recent years there is increased demand for better quality images in various applications such as biomedical imaging, surveillance and video enhancement. Image enhancement is also widely useful for satellite image applications which include mine detection, urban planning, military planning, intelligence and disaster monitoring/evaluation. Image resolution and contrast are the two major issues of images in these fields. Images are processed in order to enhance the quality of the digital images.

An image is a picture or photograph and is usually a numeric representation of a two dimensional function. An image is represented by intensity at a particular point whose spatial coordinates are on generally $\mathrm{x}$ and $\mathrm{y}$ axis coordinates. It is a two dimensional collection of pixel values. The resolution or dimension is often used for a pixel count in digital imaging. In this technique the image is enhanced from a lower resolution (dimension) to a higher resolution (dimension). Contrast is the difference in luminance that makes an object distinguishable. In visual perception of real world, contrast is determined by the difference in color and brightness of the object and the other object within the same field of view. Because the human visual system is more sensitive to contrast than absolute luminance, we can perceive the world similarly regardless of the huge changes in illumination. In this technique, the contrast is enhanced by changing the illumination information of the image.

One of the commonly used techniques for image resolution enhancement is interpolation. Interpolation increases the number of pixels in the digital image. The image interpolation algorithms widely used are bi-cubic interpolation [1], bilinear interpolation, nearest neighbor interpolation and wavelet zero padding [2].

Discrete Wavelet Transform (DWT), Stationary wavelet transform (SWT) and integer wavelet transform [3] are three versatile tools for modern image processing. These techniques have various image processing applications such as super resolution [4]-[9], facial reconstruction [10], multiple description coding [11] and video enhancement [12].

In the proposed technique bi-cubic interpolation is used. Bicubic interpolation looks best with smooth edges and less blurring compared to the other techniques. In the proposed technique SWT and IWT are the two methods used to preserve the high frequency components of the digital image. Integer wavelet transform using lifting scheme is an efficient algorithm to calculate wavelet transform. Also integer wavelet transform using lifting schemes provides a perfect reconstruction.

Low contrast image results due to low light conditions, lack of dynamic range of the camera sensor. Contrast stretching operation results in good quality image. For this, there have been several methods such as general histogram equalization (GHE) [13], Dynamic histogram equalization (DHE) [14], Dynamic Histogram Specification (DHS) [15], Brightness preserved dynamic histogram equalization [16].GHE is a common technique for enhancing the appearance of the image. It involves finding a grey scale transformation function that creates an output image with a uniform histogram.DHE obtained from dynamic histogram specification generates the specified histogram dynamically from the input image. In the proposed technique image contrast enhancement is done using singular value based image equalization. Here it equalizes the singular value matrix obtained from singular value decomposition.

The proposed technique is compared with other resolution enhancement methods such as

-Wavelet zero padding [WZP]. [2]

-DWT based super resolution [DWT SR]. [4]

-DWT-SWT based super resolution [DWT-SWT SR]. [5] 
In wavelet zero padding[WZP], the discrete wavelet transform is applied on the low resolution image to decompose it into four sub bands in different frequencies (LL,LH,HL,HH).The unknown coefficients in the LH and HL sub band are replaced with zeros. The HH sub band is set with all zeros. Inverse transform on the sub bands gives the desired up sampled image.

In DWT based super resolution [DWT SR], the interpolated high frequency sub bands and low resolution images are used. Inverse DWT (IDWT) has been applied to combine all these images to obtain the final output.

In SWT-DWT based [DWT-SWT SR] method the image resolution enhancement is based on interpolating the high frequency sub bands obtained using discrete wavelet transform and the input image. Edges are enhanced by using stationary wavelet transform. Inverse discrete wavelet transform combines all the sub images to obtain the final output.

\section{PROPOSED IMAGE ENHANCEMENT}

The proposed enhancement technique includes two steps. Image resolution enhancement is done as first step. Contrast enhancement is done in the second step.

\subsection{Image Resolution Enhancement}

In order to enhance the resolution of the image the high frequency components are corrected using stationary wavelet transform and singular value decomposition. Initially the input low resolution image of dimension $(m \times n)$ is decomposed into four sub band images (LL, LH, HL, HH) using stationary wavelet transform. The three high frequency sub bands (LH, $\mathrm{HL}$, and $\mathrm{HH}$ ) contain the high frequency components of the input image. In parallel, the singular value decomposition is applied on input low resolution image. The singular value decomposition factorizes the input image I into a matrix, which is of the form,

$$
I=U_{I} \Sigma_{I} V_{I}^{\prime}
$$

Where $U_{I}$ and $V_{I}$ are orthogonal square matrices known as hanger and aligner respectively. The matrix, $\Sigma_{I}$ contains the intensity information of the given image. The orthogonal matrices contain elements with high frequency values. The high frequency sub bands obtained using stationary wavelet transform are multiplied with the orthogonal matrix coefficients. Further it is added with the same high frequency components to obtain the estimated coefficients. The resulting three high frequency sub band images are interpolated using bi-cubic interpolation. The low resolution input image is also interpolated. By interpolating the input image and high frequency sub bands with a factor of 2 , the up scaled sub images become a size of $(2 m \times 2 n)$. Finally the corrected sub bands and the interpolated input images are combined using inverse integer wavelet transform to get a high resolution image of dimension $(4 m \times 4 n)$.

\subsection{Image Contrast Enhancement}

A combination of singular value decomposition and integer wavelet transform is used for contrast enhancement. The singular value decomposition factorizes the image matrices and the singular value matrix contains the intensity information. Therefore changing the singular value will directly affect the illumination of the image. In the proposed technique, Integer wavelet transform is applied on the high resolution output image, to decompose it into four sub band images $\left(L L_{I}, L H_{I}, H L_{I}, H H_{I}\right)$. The LL sub band image contains all illumination information. So manipulating the LL sub band gives the enhancement in contrast. In parallel, the high resolution image is processed using general histogram equalization (GHE) to generate an image $\hat{\mathrm{I}}$. The histogram equalized image is further decomposed into four sub band images $\left(L L_{\hat{l}}, L H_{\hat{l}}, H L_{\hat{l}}, H H_{\hat{I}}\right)$ using integer wavelet transform with lifting scheme. Then, singular value decomposition is applied on both of the images $L L_{I}$ and $L L_{\hat{I}}$ to get two matrices, $L L_{I}=U_{L L I} \Sigma_{L L I} V_{L L I}{ }^{\prime}$ and $L L_{\hat{I}}=U_{L L \hat{I}} \Sigma_{L L \hat{I}} V_{L L \hat{I}}{ }^{\prime}$.

The correction coefficient matrix of the singular value matrix is calculated by using the equation,

$$
\zeta=\frac{\max \left(\sum L L I\right)}{\max \left(\sum L L \hat{I}\right)}
$$

Where $\Sigma_{L L I}$ is the LL singular value matrix of the input image and $\Sigma_{L L \hat{I}}$ is the LL singular value matrix of the output of GHE.A new LL image, $\bar{\Sigma}_{L L I}$, has been constructed by ,

$$
\bar{\Sigma}_{L L I}=\zeta \Sigma_{L L I}
$$

Where $\bar{\Sigma}_{L L I}$ is referred as the singular value matrix of the equalized image. Using $\bar{\Sigma}_{L L I}$ a new equalized LL sub image matrix is formed. The new equalized LL sub image, $\overline{L L}_{I}$, is given by the equation,

$$
\overline{L L}_{I},=U_{L L I} \bar{\Sigma}_{L L I} V_{L L I}^{\prime}
$$

Now the equalized image is reconstructed by applying inverse integer wavelet transform on the sub bands $\left(\overline{L L}_{I}, L H_{I}, H L_{I}, H H_{I}\right)$.

Finally, bi-cubic interpolation is done to further increase the resolution.

Figure 1 explains the major steps involved in the proposed technique. A low resolution image with poor resolution and poor illumination is used as input. The steps in Fig. 1 are carried out to get an image enhanced in resolution and contrast.

\section{RESULTS AND DISCUSSIONS}

The proposed technique has been tested on different satellite images. Figures 2-4 show the output images obtained using proposed method and other resolution enhancement technique such as WZP, DWT SR, and SWT-DWT SR. It can be observed that there is a significant enhancement in resolution and contrast of the proposed method compared to other methods.

When image enhancement techniques are used as preprocessing tools for other image processing techniques, then quantitative measures can determine which techniques are most appropriate. So, in addition to the visual comparison, the performance has also been compared using Peak-Signal-toNoise Ratio (PSNR). PSNR can be obtained by using the following formula,

$$
P S N R=10 \log _{10}\left(\frac{R^{2}}{M S E}\right)
$$


Where $R$ is the maximum pixel value of the input image (for 8 -bit image $\mathrm{R}=255$ ).MSE is the mean square error. It is given by the equation,

$$
M S E=\frac{\sum_{i, j}\left(I_{\text {in }}(i, j)-I_{\text {out }}(i, j)\right)^{2}}{M \times N}
$$

Where $I_{\text {in }}$ the low resolution is input image and $I_{\text {out }}$ is the output image. $M$ and $N$ are the size of the images.

Tables 1 and Table 2 show the comparison between the proposed method and the other methods, such as WZP, DWT SR, DWT-SWT SR using PSNR values and MSE values respectively. The results show the proposed technique outperforms the other methods.

Table 3 shows the entropy values. Entropy can be used to characterize the texture of the image. The entropy values of the output images of the proposed technique are very close to the entropy values of the input images. Thus the result shows the output image of the proposed method contain most of the information content in the input image.

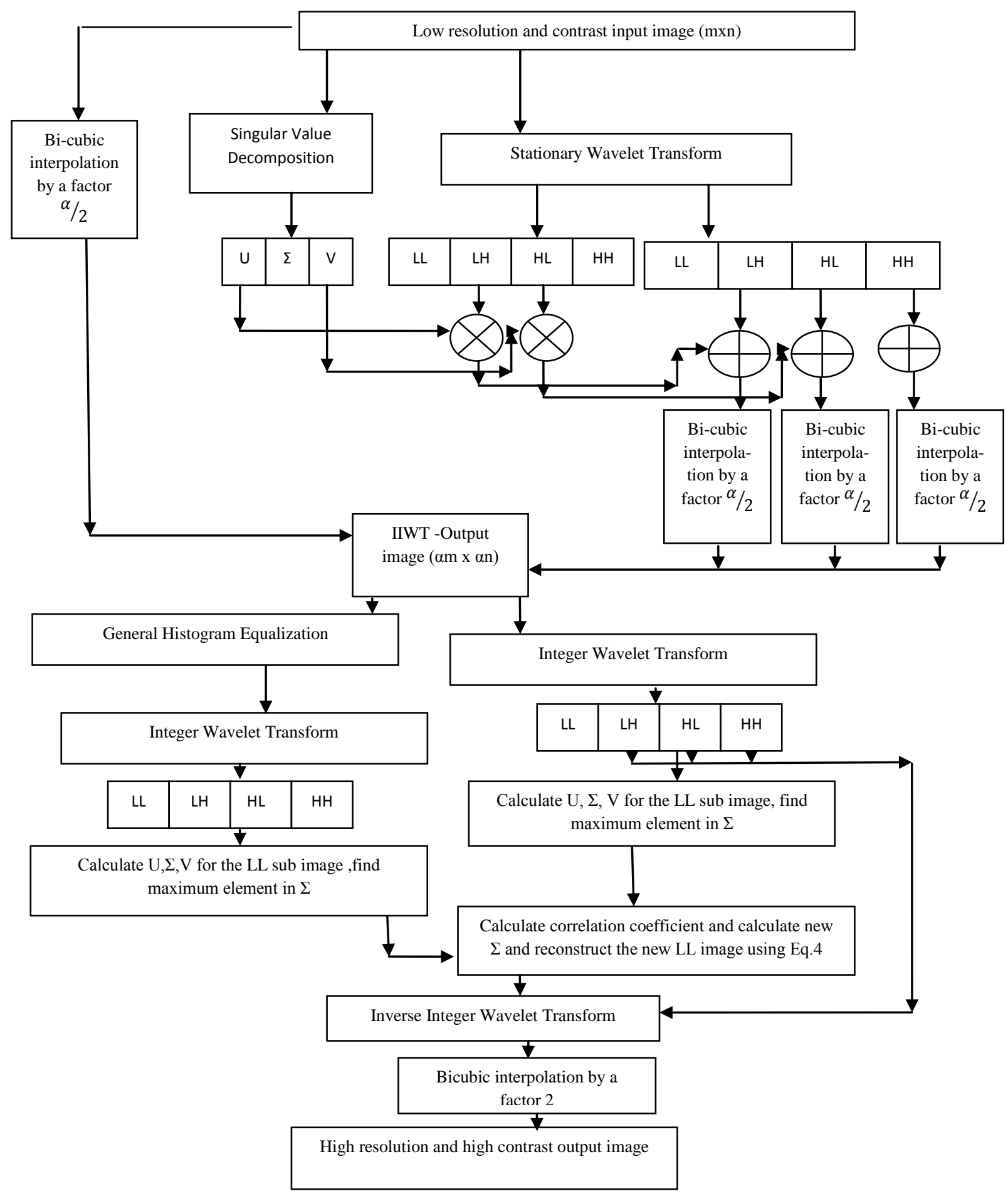

Fig 1: Algorithm of proposed enhancement technique. 


\subsection{Output Figures}

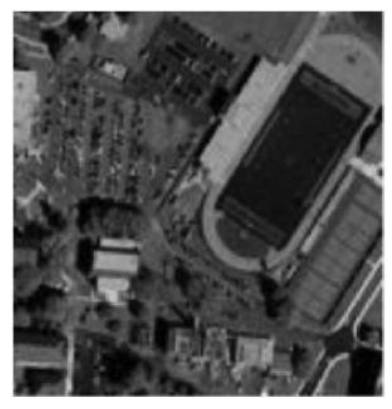

(a)

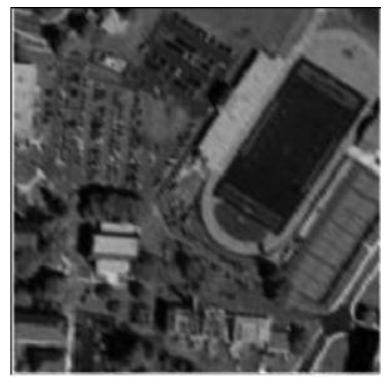

(b)

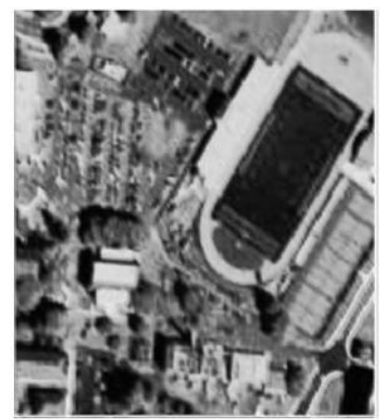

(c)

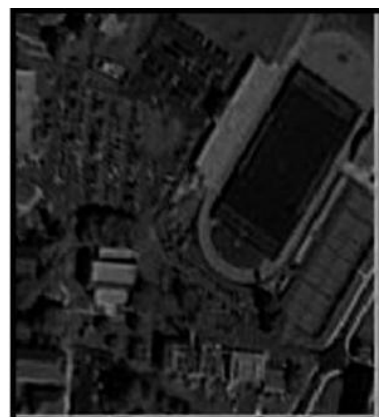

(d)

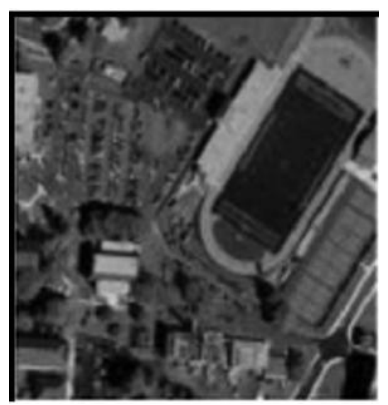

(e)

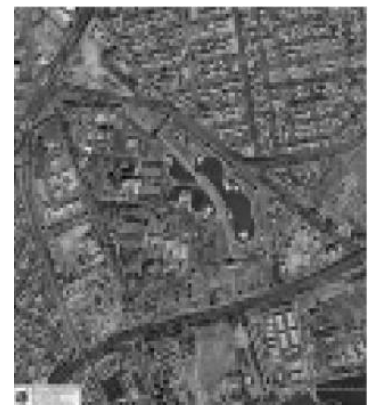

(a')

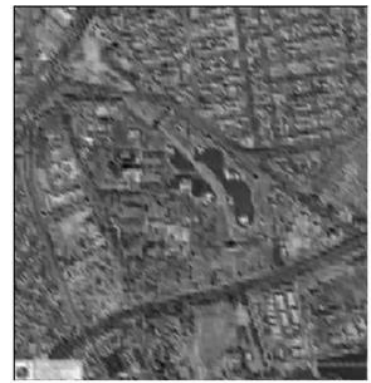

(b')

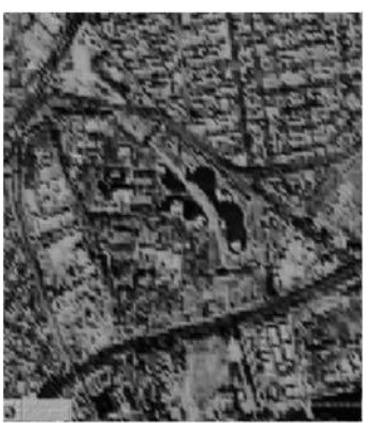

(c')

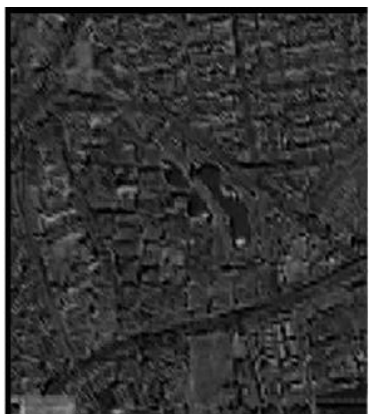

(d')

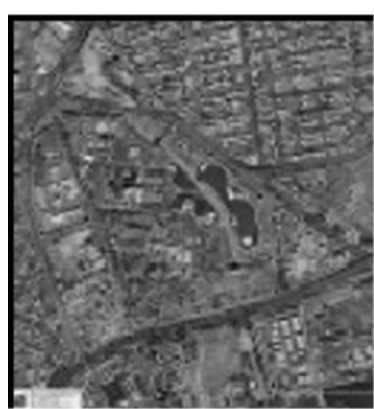

(e')

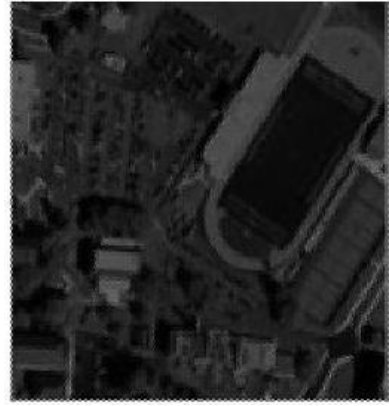

(f)

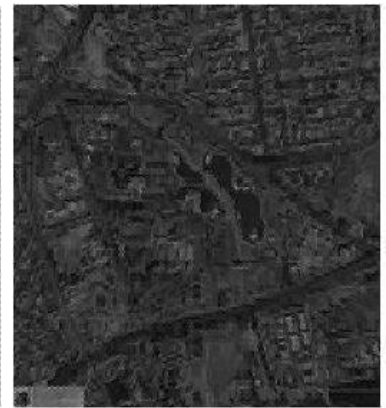

(f')
Fig. 2-1, 2-2 (a and $\left.a^{\prime}\right)$ low resolution input images, (b and b') high resolution output images using proposed technique with enlargement from $256 \times 256$ to $1024 \times$ 1024 . (c and $\left.c^{\prime}\right)$ high resolution and contrast images using proposed technique. ( $d$ and $d^{\prime}$ ) enhanced images using swt-dwt based technique. (e and $e^{\prime}$ ) enhanced images using dwt based method. (f and $f^{\prime}$ ) enhanced images using WZP.

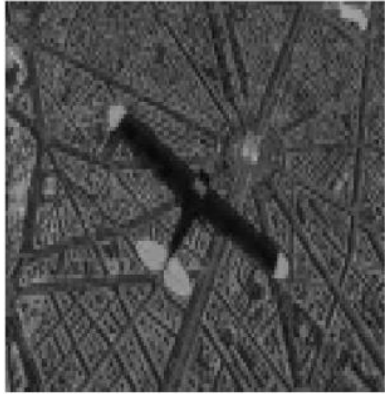

(a)

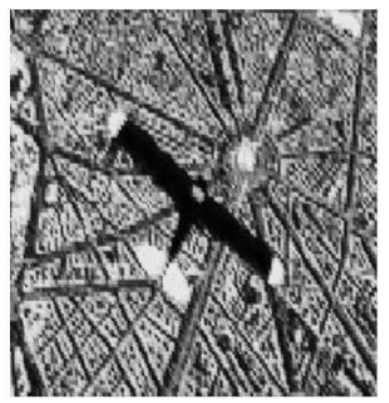

(c)

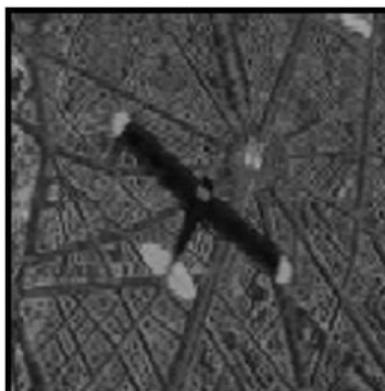

(e)

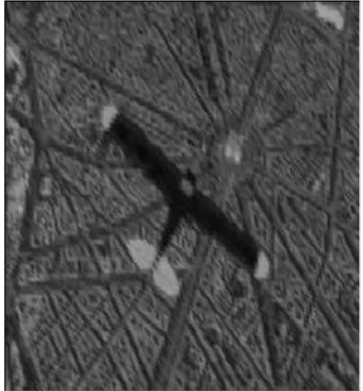

(b)

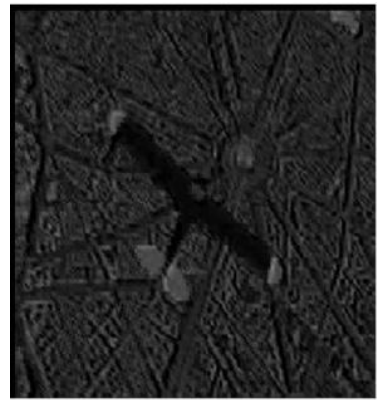

(d)

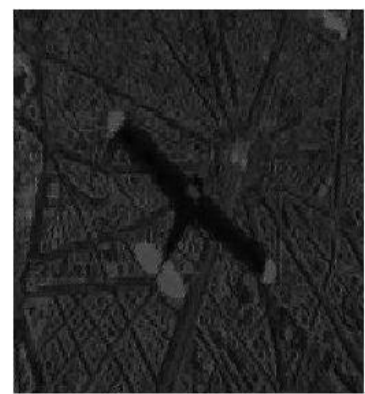

(f)
Fig. 3 (a) low resolution input image, (b) high resolution output image using proposed technique with enlargement from $256 \times 256$ to1024 $\times 1024$. (c) High resolution and contrast image using proposed technique. (d) enhanced image using swt-dwt based technique. (e) enhanced image using dwt based method. (f) enhanced image using WZP. 


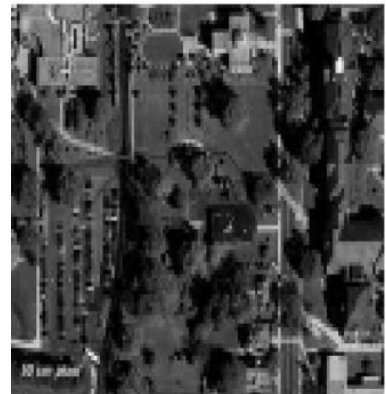

(a)

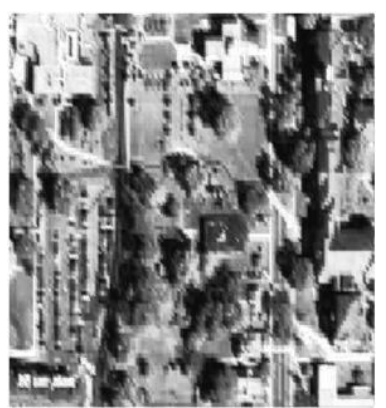

(c)

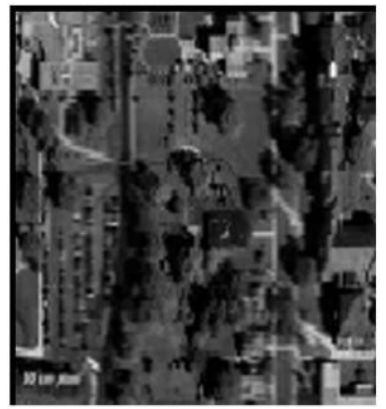

(e)

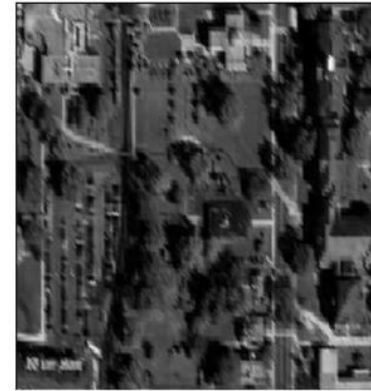

(b)

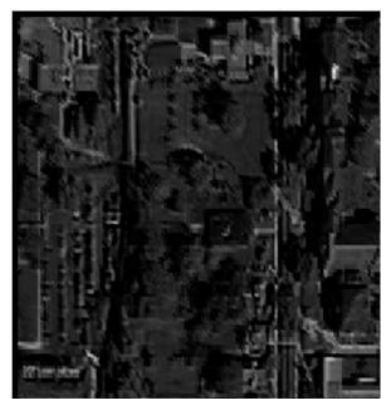

(d)

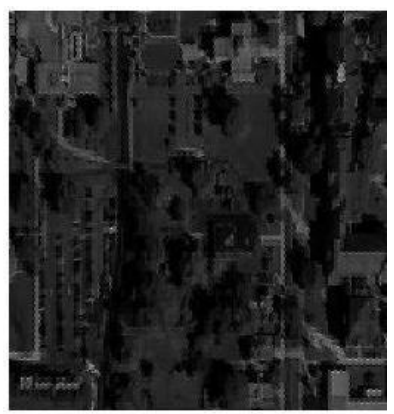

(f)
Fig. 4 (a) low resolution input image, (b) high resolution output image using proposed technique with enlargement from $256 \times 256$ to1024 $\times 1024$. (c) high resolution and contrast image using proposed technique.(d) enhanced image using swt-dwt based technique. (e) enhanced image using dwt based method. (f) enhanced image using WZP.

Table 1. PSNR results for resolution enhancement from a size of $256 \times 256$ to $1024 \times 1024$ for the proposed technique compared with some other methods.

\begin{tabular}{|c|c|c|c|c|}
\hline \multicolumn{5}{|c|}{ PSNR $(\boldsymbol{d B})$} \\
\hline $\begin{array}{c}\text { Image } \\
\text { /Method }\end{array}$ & Fig.2-1 & Fig.2-2 & Fig.3 & Fig.4 \\
\hline WZP & 24.06 & 24.67 & 24.19 & 24.08 \\
\hline DWT SR & 29.34 & 28.04 & 27.29 & 28.02 \\
\hline $\begin{array}{c}\text { SWT-DWT } \\
\text { SR }\end{array}$ & 24.06 & 24.72 & 24.26 & 24.07 \\
\hline $\begin{array}{c}\text { PROPOSED } \\
\text { METHOD }\end{array}$ & 39.17 & 32.93 & 30.94 & 37.02 \\
\hline
\end{tabular}

Table 2. MSE results for resolution enhancement from a size of $256 \times 256$ to $1024 \times 1024$ for the proposed technique compared with some other methods.

\begin{tabular}{|c|c|c|c|c|}
\hline \multicolumn{5}{|c|}{ MSE } \\
\hline $\begin{array}{c}\text { Image } \\
\text { /Method }\end{array}$ & Fig.2-1 & Fig.2-2 & Fig.3 & Fig.4 \\
\hline WZP & 226.32 & 254.37 & 247.64 & 204.87 \\
\hline DWT SR & 99.8 & 112.61 & 108.16 & 100.64 \\
\hline $\begin{array}{c}\text { SWT-DWT } \\
\text { SR }\end{array}$ & 220.14 & 250.91 & 240.39 & 198.19 \\
\hline $\begin{array}{c}\text { PROPOSED } \\
\text { METHOD }\end{array}$ & 28.23 & 40.22 & 32.57 & 34.03 \\
\hline
\end{tabular}

Table 3. Entropy results for resolution enhancement from a size of $256 \times 256$ to $1024 \times 1024$ for the proposed technique compared with some other methods.

\begin{tabular}{|c|c|c|c|c|}
\hline \multicolumn{5}{|c|}{ ENTROPY } \\
\hline $\begin{array}{c}\text { Image } \\
\text { Method }\end{array}$ & Fig.2-1 & Fig.2-2 & Fig.3 & Fig.4 \\
\hline $\begin{array}{c}\text { Input low } \\
\text { resolution } \\
\text { image }\end{array}$ & 7.14 & 6.79 & 6.52 & 7.16 \\
\hline WZP & 6.28 & 5.9 & 5.61 & 6.19 \\
\hline $\begin{array}{c}\text { DWT SR } \\
\text { SWT-DWT } \\
\text { SR }\end{array}$ & 6.28 & 6.21 & 5.96 & 6.89 \\
\hline $\begin{array}{c}\text { PROPOSED } \\
\text { METHOD }\end{array}$ & 7.2 & 6.87 & 6.6 & 7.14 \\
\hline
\end{tabular}

\section{CONCLUSION}

The proposed method describes a new technique in image enhancement. The technique enhances the image and uncovers the hidden details in the image. For resolution enhancement, SWT decomposes the image into sub bands. The high frequency sub bands are corrected by singular value matrix and SWT. The inverse integer wavelet transform perfectly reconstructs the sub bands to get the high resolution image. For contrast enhancement, the sub bands obtained using integer wavelet transform is updated with the singular value matrix of the LL sub band. The inverse integer wavelet transform produces a better equalized image. The proposed method is tested on different images which show good result in PSNR, entropy and MSE. The quantitative result supports the visual results that the quality and information content of the equalized and enhanced images are better preserved through the proposed method.

\section{REFERENCES}

[1] Robert G Key, Cubic convolution interpolation for digital image processing, IEEE transactions on acoustics, speech and signal processing, vol-29, no.6, December 1981.

[2] Tinku Acharya, Pin-Sing Tsai,"Computational Foundations of Image Interpolation Algorithms", ACM Ubiquity Vol.8 2007. 
[3] Geert Uytterhoeven, Dirk Roose, Adhemar Bultheel, Integer Wavelet Transforms using the Lifting Scheme,CSCC'99 Proceedings.

[4] Hasan Demirel and Gholamreza Anbarjafari, "Discrete Wavelet Transform-Based Satellite Image Resolution Enhancement", IEEE transactions on geosciences and remote sensing, June 2011.

[5] Hasan Demirel and Gholamreza Anbarjafari, "Image Resolution Enhancement by Using Discrete and Stationary Wavelet Decomposition" IEEE transactions on Image Processing, VOL. 20, NO. 5, May2011.

[6] A. Temizel and T. Vlachos," Image resolution up scaling using directional cycle spinning," J. Electron. Imaging, vol. 14, 2005.

[7] Y. Piao, L. Shin, and H. W. Park, "Image resolution enhancement using inter-sub band correlation in wavelet domain,”, in Proc. IEEE ICIP, 2007.

[8] H. Demirel and G. Anbarjafari, "Satellite image resolution enhancement using complex wavelet transform," IEEE Geosciences and Remote Sensing Letter, vol. 7 , no. 1 .

[9] S.G. Mallat. A theory for Multiresolution signal decomposition: the wavelet representation. IEEE Transactions on Pattern Analysis and Machine Intelligence, 11(7):674-673, 2002.

[10] T. Celik, C. Direkoglu, H. Ozkaramanli, "Region-based super-resolution aided facial feature extraction from low resolution video sequences," in Proc. IEEE ICASSP, Mar. 2005
[11] Y. Rener, J. Wei, and C. Ken, "Down sample-based multiple description coding and post-processing of decoding," in Proc. 27th CCC, Jul. 16-18, 2008, pp. 253-256.

[12] H. Demirel, G. Anbarjafari, and S. Izadpanahi, "Improved motion-based localized super resolution technique using discrete wavelet transform for low resolution video enhancement," in Proc. 17th EUSIPCO, Aug. 2009.

[13] T. Kim, H.S. Yang, "A Multidimensional Histogram Equalization by Fitting an Isotropic Gaussian Mixture to a Uniform Distribution", IEEE International Conference on Image Processing, 8-11 Oct. 2006, pp: 2865 - 2868

[14] A. R. Weeks, L. J. Sartor, and H. R. Myler, 'Histogram specification of 24-bit color images in the color difference (C-Y) color space', Proc. SPIE, 1999, 3646 , pp. 319-329.

[15] M. Abdullah-Al-Wadud, H. Kabir, M. A., A.Dewan, C. Oksam, "A Dynamic Histogram Equalization for Image Contrast Enhancement”, ICCE 2007. 10-14 Jan. 2007.

[16] H. Ibrahim and N. S. P. Kong, "Brightness preserving dynamic histogram equalization for image contrast enhancement," IEEE Trans. Consum. Electron. vol. 53, no. 4, pp. 1752-1758, Nov. 2007.

[17] R. C Gonzalez and R. E Woods, Digital Image Processing. Eaglewood Cliffs, NJ: Prentice-Hall. 2007. 